\title{
Dense spray evaporation as a mixing process
}

\author{
A. de Rivas ${ }^{1}$ and E. Villermaux ${ }^{1,2}$ \\ ${ }^{1}$ Aix Marseille Université, CNRS, École Centrale de Marseille, IRPHE, UMR No. 7342, \\ 13384 Marseille, France \\ ${ }^{2}$ Institut Universitaire de France, 75005 Paris, France \\ (Received 12 January 2016; published 18 May 2016)
}

\begin{abstract}
We explore the processes by which a dense set of small liquid droplets (a spray) evaporates in a dry, stirred gas phase. A dense spray of micron-sized liquid (water or ethanol) droplets is formed in air by a pneumatic atomizer in a closed chamber. The spray is conveyed in ambient air as a plume whose extension depends on the relative humidity of the diluting medium. Standard shear instabilities develop at the plume edge, forming the stretched lamellar structures familiar with passive scalars. Unlike passive scalars however, these lamellae vanish in a finite time, because individual droplets evaporate at their border in contact with the dry environment. Experiments demonstrate that the lifetime of an individual droplet embedded in a lamellae is much larger than expected from the usual $d^{2}$ law describing the fate of a single drop evaporating in a quiescent environment. By analogy with the way mixing times are understood from the convection-diffusion equation for passive scalars, we show that the lifetime of a spray lamellae stretched at a constant rate $\gamma$ is $t_{v}=\frac{1}{\gamma} \ln \left(\frac{1+\phi}{\phi}\right)$, where $\phi$ is a parameter that incorporates the thermodynamic and diffusional properties of the vapor in the diluting phase. The case of time-dependent stretching rates is examined too. A dense spray behaves almost as a (nonconserved) passive scalar.
\end{abstract}

DOI: 10.1103/PhysRevFluids.1.014201

\section{INTRODUCTION}

Dense sprays are, in nature and artifacts, the rule rather than exceptions. In fog and clouds [1,2], sea spray [3], liquid propellant combustion engines or synthetic atomizers [4], agricultural sprays [5], cooling towers, and even human violent respiratory events [6], droplets are formed and evolve in the vicinity of each other, influencing mutually their dynamics of evaporation $[7,8]$. This is true not only for sprays evaporating in open air or in confined chambers since the fact that the rate of evaporation of an individual droplet is influenced by the vapor concentration in the diluting environment is also known for drops sitting on a solid surface [9-11]. This fact obviously reflects the diffusive nature of the vapor flux leaving the liquid droplet towards the diluting gas: If the environment is as concentrated in vapor as in the droplet surface, no evaporation occurs.

The corresponding effect is well accounted for quantitatively at the scale of a single droplet evaporating in a quiescent environment by the celebrated $d^{2}$ law [12-15], which describes how, because the vapor gradient at the droplet surface steepens as its radius decays, the droplet diameter diminishes in a catastrophic way, going to zero in a finite time $t_{1}$. Solving the Stefan problem around a spherical droplet [16] provides

$$
t_{1}=\mathcal{K} \frac{d^{2}}{D}
$$

with

$$
\mathcal{K}^{-1}=8 \frac{\rho_{a}}{\rho_{l}} \ln (1+\mathcal{B}), \quad \mathcal{B}=\frac{\rho_{s}-\rho_{\infty}}{\rho_{a}-\rho_{s}} \ll 1,
$$

so that

$$
t_{1} \approx \frac{d^{2}}{8 D} \frac{\rho_{l}}{\rho_{s}-\rho_{\infty}}
$$


if $d$ is its initial diameter and $\rho_{s}$ and $\rho_{\infty}$ are the densities of vapor at the droplet surface and far away from it, both usually much smaller than the density of the ambient gas environment $\rho_{a}$. The density of the liquid is $\rho_{l}$ and the vapor diffuses in the gas with coefficient $D$. The above law, rooted in the spherical geometry for which the rate of evaporation is inversely proportional to the current droplet radius (or pellet, which was actually discovered with the sublimation of solid iodine crystals [17]), was soon extended to the case when the droplet is in relative motion with its environment, the vapor gradient at its surface being steepened in a boundary layer all the more thin that the motion is fast, thus hastening the process $[18,19]$.

However, whereas the isolated droplet limit, either at rest or in relative motion in the environment, is well understood and documented, the case of a collection of droplets evaporating as they move in a dry environment has been less studied [20]. Its complete description requires knowledge of both the droplet concentration and the local vapor concentration in space, rendering the task in unsteady turbulent flows extremely difficult [21]. Note that droplets may not only evaporate but, if they are sufficiently close to each other, also collide and possibly coalesce. Encounters between drops is all the more frequent in stagnation point regions of the flow that they have inertia, whose intensity is measured relative to viscous damping by the Stokes number $\mathrm{St}=\tau / \tau_{a}$, where $\tau=\left(d^{2} / \nu\right)\left(\rho_{l} / \rho_{a}\right) / 18$ is the viscous relaxation time of a droplet in an environment of viscosity $v$ and $\tau_{a}$ is a measure of the environment flow unsteadiness [22-24]. For $\mathrm{St}>1$, denser droplets are expelled from the vortical regions of the flow and aggregate in the convergent regions, while for $\mathrm{St} \ll 1$, they behave essentially as a passive tracer. Still, even in this limit, the question of a dense spray of fine droplets passively advected and evaporating in a dry diluting environment remains to be investigated.

Guided by observations, we will draw an analogy with the dynamics of passive scalars. The support of these forms stretched sheets and lamellae in random flows and it will be shown that the first stages of a plume of spray released in a stirred environment indeed falls under such a description. If $s(t)$ denotes the transverse thickness of a scalar lamella stretched in a flow, molecular mixing occurs when the rate of compression $-\dot{s} / s$ balances the rate of diffusive smoothing $D / s^{2}$ (see $[25,26]$ and references therein). For instance, in exponentially diverging flows, $s=s_{0} e^{-\gamma t}$ and the balance is attained at the mixing time

$$
t_{m}=\frac{1}{2 \gamma} \ln \mathrm{Pe} \quad \text { when } \quad \mathrm{Pe}=\frac{\gamma s_{0}^{2}}{D} \gg 1 .
$$

At large Péclet number Pe, the mixing time now scales like $\gamma^{-1}$ and is only weakly dependent on $D$, unlike $t_{1}$ in Eq. (3). Vapor diffuses, at a rate enhanced by stretching according to Eq. (4), but the droplets themselves do not, because they are typically big (non-Brownian). Droplets in a lamella of dense spray will thus evaporate at a rate prescribed by the rate at which vapor can be evacuated from the core of the lamella itself.

The present paper explores this scenario and shows from experiments how the lifetime $t_{v}$ of a dense spray lamella can be interpreted by an adapted form of Eq. (4). The precise meaning of dense is given accordingly.

\section{DENSE SPRAYS}

\section{A. Spray production and observation}

The sprays we study are produced by a pneumatic atomizer consisting in a millimetric tube whose lower extremity dips into the liquid to be atomized (water or ethanol) and the upper one over which a fast air stream blows at a right angle. The liquid ascends the tube towards the depression at the top extremity, where it is atomized into droplets of diameters $d$ of the order of a few microns. The mist thus formed recirculates into a closed chamber, so that the droplets are in equilibrium with their vapor, and is then conveyed towards the test section, a 2-m-long $10 \times 10 \mathrm{~cm}^{2}$ section channel filled with quiescent air at controlled humidity, and fitted with transparent walls, through a contracted nozzle of radius $h$, of the order of $1 \mathrm{~cm}$ (Figs. 1 and 2). 

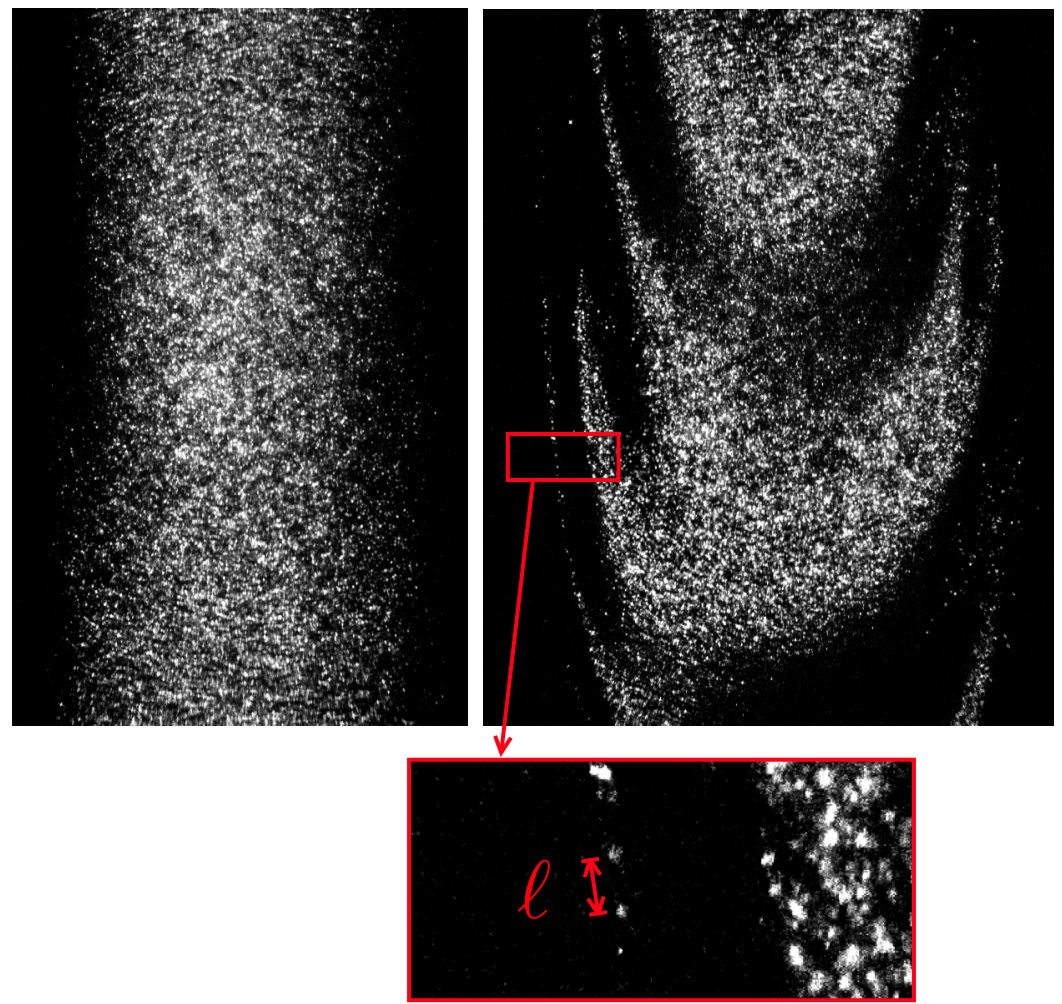

FIG. 1. Dense spray of water droplets as it exits a tube in the visualization channel (left) and farther downstream as is has developed a shear instability with the surrounding air (right). The jet radius is $h=5 \mathrm{~mm}$ and $\mathrm{Re}=700$. The droplets, a few microns in diameter (see Fig. 3), are separated by a typical distance $\ell \lesssim 10 d$ in the spray, while this distance increases at the very end of the spray lamellae evaporation (see Sec. IV B). The top left image is $1.85 \mathrm{~cm}$ wide.

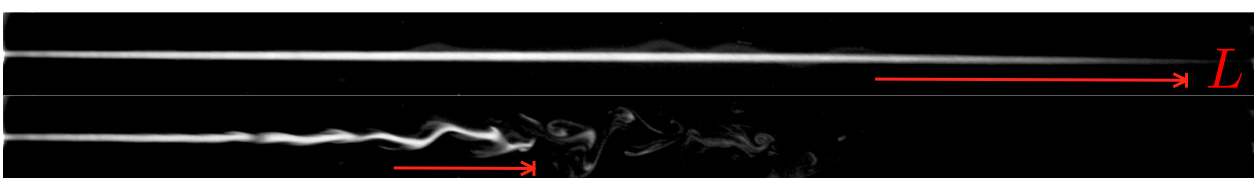

(a)

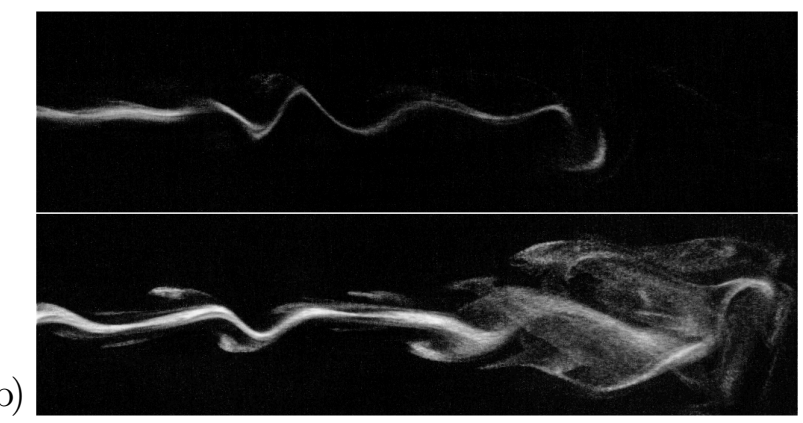

FIG. 2. (a) A laminar water spray jet $(h=5 \mathrm{~mm}$ and $\mathrm{Re}=200)$ in air at relative humidity $\rho_{\infty} / \rho_{s}=0.5$ is perturbed at the inlet by a small sinusoidal perturbation, triggering varicose instabilities that shorten drastically the spray length $L$. (b) Two transitional jets $\left(h=5 \mathrm{~mm}\right.$ and $\left.\operatorname{Re} \gtrsim 10^{3}\right)$ of water sprays in dry (top) (relative humidity $\left.\rho_{\infty} / \rho_{s}=0.3\right)$ and humid (bottom) $\left(\rho_{\infty} / \rho_{s}=0.9\right)$ air environments. 
TABLE I. Physical properties of the fluids at $T=293 \mathrm{~K}$. The density of the liquid is $\rho_{l}$ and $M$ is the molecular mass. The saturation density derives from the vapor pressure as $\rho_{s}=M p_{s} / R T$ with $R=8.31 \mathrm{~J} / \mathrm{mol} \mathrm{K}$. The diffusion coefficients $D$ refer to diffusion in air.

\begin{tabular}{lccccc}
\hline \hline Fluid & $\rho_{l}\left(\mathrm{~kg} \mathrm{~m}^{-3}\right)$ & $p_{s}(\mathrm{~Pa})$ & $M\left(\mathrm{~kg} \mathrm{~mol}^{-1}\right)$ & $\rho_{s}\left(\mathrm{~kg} \mathrm{~m}^{-3}\right)$ & $D\left(\mathrm{~m}^{2} \mathrm{~s}^{-1}\right)$ \\
\hline water & 1000 & 2340 & $18 \times 10^{-3}$ & 0.017 & $2.8 \times 10^{-5}$ \\
ethanol & 789 & 7410 & $46 \times 10^{-3}$ & 0.13 & $1.3 \times 10^{-5}$ \\
\hline \hline
\end{tabular}

The top-hat velocity profile has velocity $u$, ranging from 0.1 to $10 \mathrm{~m} / \mathrm{s}$, so the jet Reynolds number $\operatorname{Re}=u h / v$ ranges from about $10^{2}$ to $10^{4}$ with $v=1.5 \times 10^{-5} \mathrm{~m}^{2} / \mathrm{s}$ the viscosity of air. Unsteadiness in the flow arises from the development of shear instabilities with time scale $\tau_{a} \sim h / u$. The corresponding Stokes number $\mathrm{St}=\left(u d^{2} / \nu h\right)\left(\rho_{l} / \rho_{a}\right) / 18$ ranges from $10^{-4}$ to $10^{-2}$ in air $\left(\rho_{a}=\right.$ $1.2 \mathrm{~kg} \mathrm{~m}^{-3}$ ), suggesting that the droplet field is fairly passively advected.

The resulting spray jet is illuminated along its axis by a thin uniform laser sheet encompassing typically tens of droplet interdistances $\ell$, which is such that $\ell / d \lesssim 10$. The corresponding liquid volume fraction is $\varphi \sim(d / \ell)^{3}$, giving a net density of liquid (density $\rho_{l}$ ) plus vapor (saturation density $\rho_{s}$ ) such that $\rho=\varphi \rho_{l}+(1-\varphi) \rho_{s}$ in the spray. That density is typically slightly larger than the density of the ambient air $\rho_{a}$, so the (noticeable) buoyancy effects however were not dominant in these experiments.

Depending on the type of measurement, the spray is imaged either in focus at a right angle from the laser sheet to document the flow dynamics (as in Fig. 1) or at a larger angle in a defocused fashion to study droplet sizes (see Sec. II C). The physical properties of the fluids relevant to this study are listed in Table I.

\section{B. Influencing factors}

Being projected into a dryer atmosphere, the vapor in the saturated spray jet diffuses in the environment, thus lowering the vapor concentration in the jet and therefore allowing for droplets to evaporate. As long as the jet is laminar $\left(\operatorname{Re} \lesssim 10^{3}\right.$ ) (see [27]), diffusion is purely radial, with a vapor mass flux $D\left(\rho_{s}-\rho_{\infty}\right) / \delta(z)$ oriented in the plane perpendicular to the direction $z$ of the jet axis, through a laminar boundary layer $\delta(z) \sim \sqrt{D z / u}$. All the droplets have evaporated, defining the spray jet length $L$ [Fig. 2(a)] when the injected mass $\sim \rho h^{2} u$ has crossed the boundary layer, which is for

$$
\frac{L}{h} \sim \operatorname{ReSc}\left(\frac{\rho}{\rho_{s}-\rho_{\infty}}\right)^{2},
$$

where $\mathrm{Sc}=v / D$ is the Schmidt number. This relation, which is of a Burke-Schumann type [28], is familiar in convection-diffusion problems as those encountered with laminar flames and can be altered to account for buoyancy forces [29].

However, when this equilibrium is even slightly perturbed by, for instance, a small sinusoidal perturbation at the orifice triggering jet instabilities, the length $L$ shortens drastically, as shown in Fig. 2(a). The reason, analyzed specifically in the following, is that the jet, now destabilizing, is stretched, steepening the vapor boundary layer at its periphery, thus enhancing the vapor diffusive flux.

A way to understand the scaling structure of Eq. (5) is to balance the transit time $L / u$ with the diffusion time of the vapor across the jet $h^{2} / D$, giving indeed $L / h \sim \operatorname{Re} \mathrm{Sc}$, namely, a jet length of the order of a few hundred jet radii, as shown in Fig. 2(a). This balance also suggests that if the vapor is extracted from the jet core within a time $t_{v}$ shorter than the pure diffusion time, then the length $L=u t_{v}$ will be reduced. Hastening diffusion, through substrate unsteadiness, shortens the jet.

Another trivial factor influencing the spray extent is the ambient concentration of vapor $\rho_{\infty}$. For a given flow hydrodynamics [such as for the two jets in Fig. 2(b)], the extent of the spray plume 

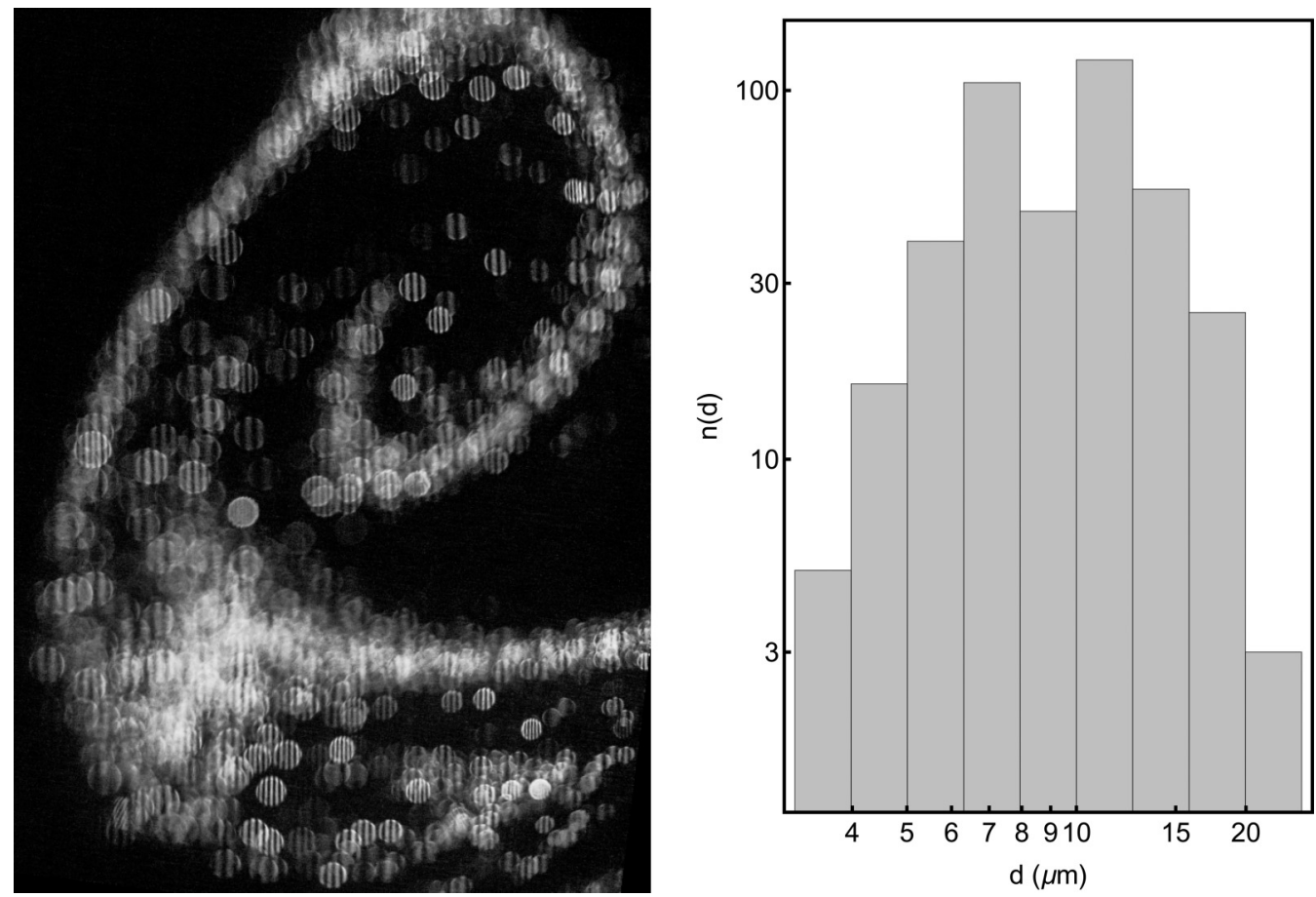

FIG. 3. Stretched spray lamella of ethanol in air seen with the IPI technique and corresponding droplet size histogram $n(d)$, obtained by measuring the patterns of about $\int n(d) d d=400$ droplets.

is larger when the environment concentration is closer to the saturation concentration $\rho_{s}$. Because diffusion is the microscopic effect at play, only concentration differences matter and the lifetime of a droplet or of a spray is infinite when $\rho_{\infty} \rightarrow \rho_{s}$.

\section{Inadequacy of the $d^{2}$ law}

We now have a closer look at the inside of the spray, using for this the interferometric particle imaging (IPI) technique (see the reviews in Refs. [30,31]). The droplets are indeed too small to be imaged directly but, precisely because they are small, the IPI technique uses their light refraction ability to measure their size. They are now visualized at an angle of the order of $160^{\circ}$ with the direction of the incoming laser light (wavelength $\lambda_{0}=488 \mathrm{~nm}$ for an argon-ion laser) and the method consists in constructing an interference pattern between a ray refracted through the liquid and a ray reflected at its surface, having thus a path-length difference function of the droplet diameter. The two rays interfere on a defocused plane in an alternating pattern of bright and dark fringes, as shown in Figs. 3 and 4. If $\xi$ denotes the interfringe distance read on a image taken a distance $\mathcal{L}$ from a spherical droplet, its diameter is given by $d=\left(\lambda_{0} \mathcal{L} / \xi\right) f\left(\theta, n_{l} / n_{a}\right)$, where $f(\cdot)$ is a function of the angle $\theta$ and of the indices of refraction of the liquid $n_{l}$ and the ambient gas $n_{a}$. The method works provided the droplets are not too concentrated in space; otherwise their patterns themselves interfere with each other, restoring a continuum, as shown in Fig. 3. Under this constraint, a drop size distribution can nevertheless be obtained.

More interesting is the possibility, using this method coupled with fast cinematography, to follow the fate of an individual droplet in a (not too) dense spray. Figure 4 shows a laminar plume of ethanol droplets ascending in air. By chance, a droplet remains inside the laser illuminating sheet from the exit of the tube, to its complete vaporization, which occurs when it reaches the top of the plume. Its diameter of $5 \mu \mathrm{m}$ initially remains constant and then abruptly decays over a time of the order of $1 \mathrm{~s}$, 


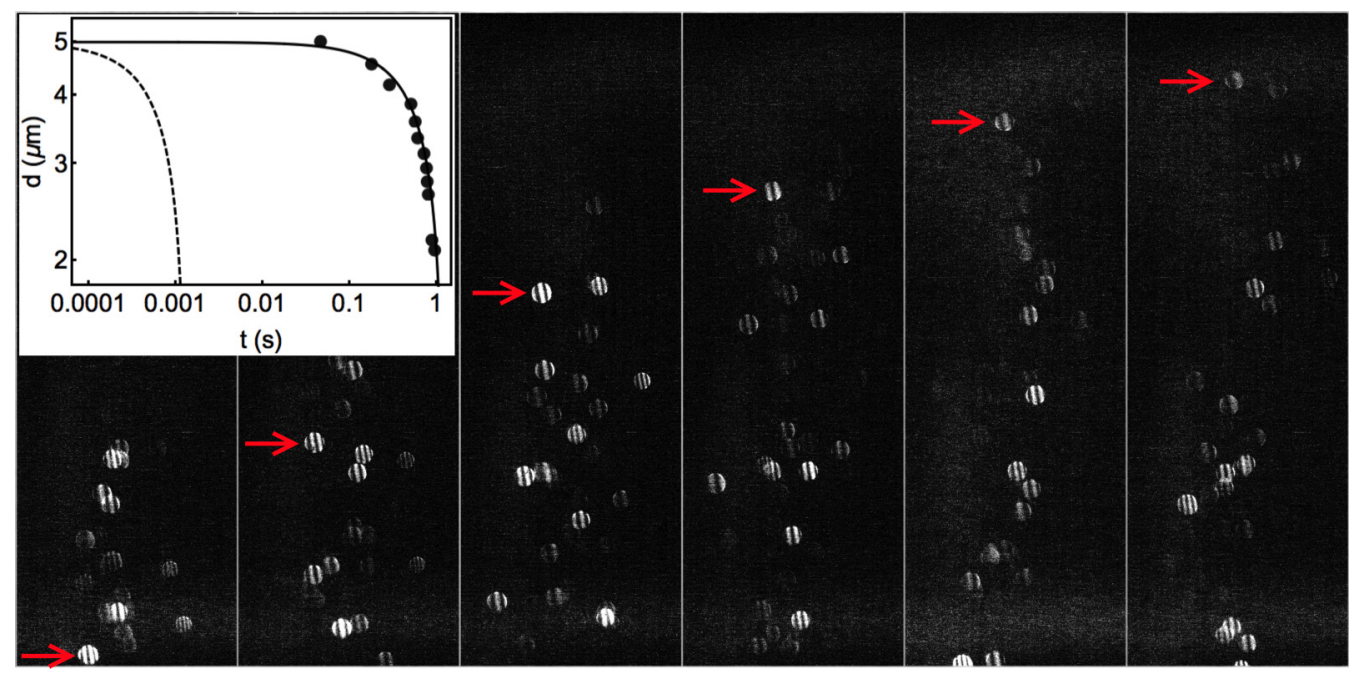

FIG. 4. Time-resolved evolution of the size of a droplet initially embedded in an ethanol spray as it evaporates in air (shown here every $\Delta t=0.18 \mathrm{~s}$ ). The inset shows the droplet diameter versus time, compared to the expectation of the $d^{2}$ law (dashed line) given its initial diameter.

as the droplet contacts the fresh air. Given its initial size and if it had been surrounded by air from the start, its lifetime would be given by Eq. (3), that is, $t_{1} \approx 10^{-3} \mathrm{~s}$. The reason why the $d^{2}$ law fails, by three orders of magnitude, at predicting its lifetime is obviously that the droplet is in equilibrium with the vapor in the spray and starts to evaporate only when the vapor has evacuated the core of the plume, which takes $1 \mathrm{~s}$. The very same phenomenon has been observed with pentane droplets trapped in a matrix of Laponite [32], where it has been measured that the droplet evaporation front penetration depth from the matrix surface is the vapor diffusion length. This observation suggests that the dynamics of droplets sizes in a dense spray is slaved at the dynamics of the vapor concentration field, thus bridging the problem of dense spray evaporation with the field of scalar mixing.

\section{STRETCHING ENHANCED EVAPORATION}

Either because it destabilizes as a whole in a sinusoidal mode (at moderate Re) or because it is peeled off at its surface by shear instabilities [at higher Re (see [27] and Fig. 6)], the spray jet ultimately shapes into a set of elongated structures, stretched in one direction as lamellae or in two directions as sheets that are, in all cases, compressed transversally (Figs. 5 and 7). We recall the dynamics of the concentration field of the vapor diffusing from a stretched structure in a dry environment and show how it can be useful at describing the evolution of regions where droplets have not evaporated yet (Fig. 5), namely, the spray thickness $s(t)$.

\section{A. Diffusion on a stretched substrate}

Let $c$ be the (molar) concentration of the vapor and $M$ its molecular weight. The saturation concentration in the core of the lamella is $c_{s}=\rho_{s} / M$ and $c_{\infty}=\rho_{\infty} / M$ is the vapor concentration in the environment. A stretched lamella aligns with the direction of the stretch so that the gradient of concentration is notable only in the compressed direction $x$ perpendicular to it (see Fig. 5 and $[25,33,34])$. If $\gamma(t)$ denotes the compression rate, the concentration $c(x, t)$ is described by

$$
\partial_{t} c-\gamma(t) x \partial_{x} c=D \partial_{x^{2}} c
$$



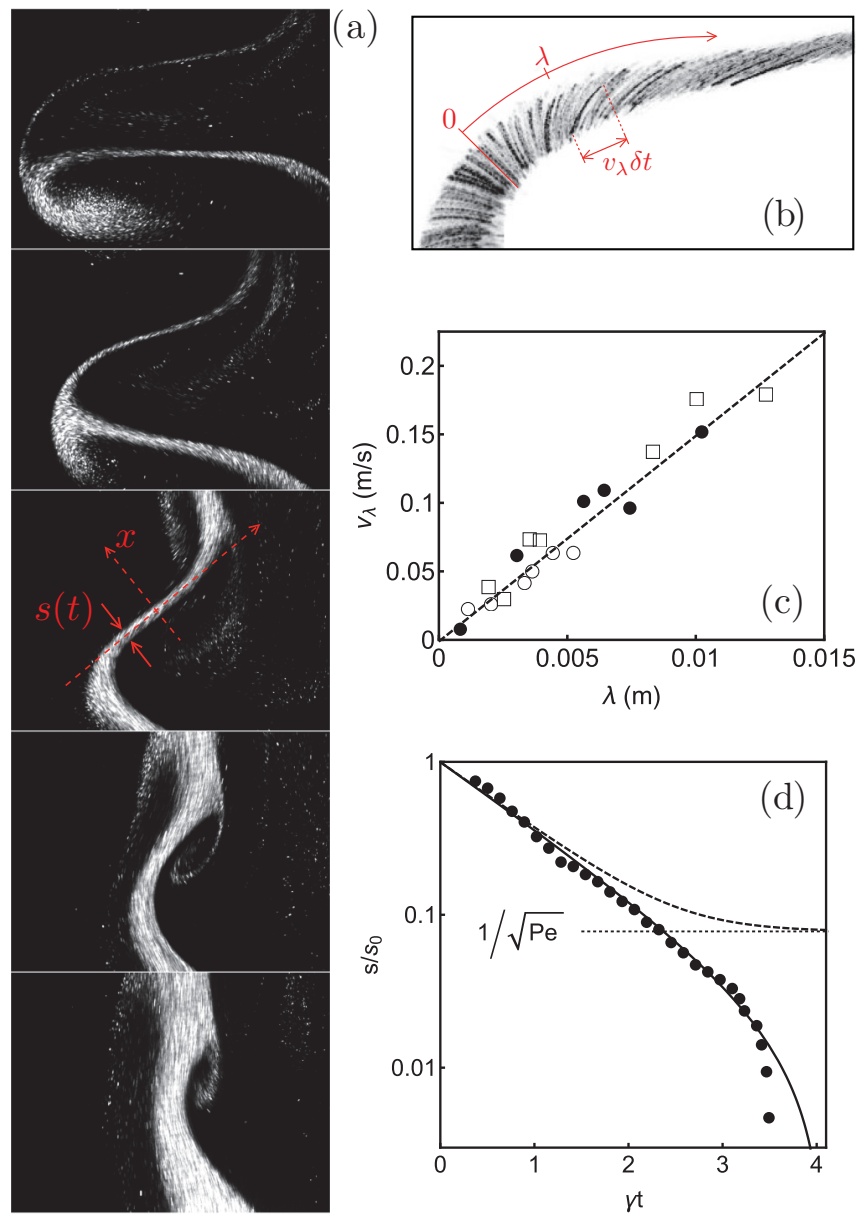

FIG. 5. (a) Sequence of a dense ethanol plume $\left(s_{0}=10 \mathrm{~mm}\right.$ and $\left.\mathrm{Re} \approx 10^{3}\right)$ undergoing a shear instability in air and destabilizing in the visualization plane, shown from bottom to top every $0.04 \mathrm{~s}$. The core of the plume stretches into a lamella, which vanishes in the dry environment. (b) Droplets trajectories revealed by the superposition of their successive locations during $\delta t=0.06 \mathrm{~s}$, identifying a stagnation point in $\lambda=0$ and an increasing velocity component $v_{\lambda}$ in the stretching direction $\lambda$ along the lamella. (c) Measurement of the stretching rate $\gamma$ such that $v_{\lambda}=\gamma \lambda$ at three different instants of time, $t=0.07 \mathrm{~s}(\circ), t=0.11 \mathrm{~s}(\bullet)$, and $t=0.15 \mathrm{~s}(\square)$, showing that $\gamma \approx 15 \mathrm{~s}^{-1}$ for all times. (d) Time evolution of the lamella thickness $s(t)$ compared to the expectation from Eq. (17) with $\mathrm{Pe}=102$ and $\phi=0.021$ (solid line). The dashed line shows the evolution of the vapor concentration profile thickness $s_{0} e^{-\gamma t} \sqrt{\left(e^{2 \gamma t}-1\right) / \mathrm{Pe}+1}$ and the dotted line corresponds to the final Batchelor scale $\sqrt{D / \gamma}=s_{0} / \sqrt{\mathrm{Pe}}$.

which, after a suitable transformation of time by ( $s_{0}$ stands for the initial lamella thickness),

$$
\tau=\frac{D}{s_{0}^{2}} \int_{0}^{t} d t^{\prime} \exp \left(2 \int_{0}^{t^{\prime}} d t^{\prime \prime} \gamma\left(t^{\prime \prime}\right)\right)
$$

and rescaling of space by $\xi=x \sqrt{\tau} / \eta$ with

$$
\eta=s_{0} \sqrt{\tau} \exp \left(-\int_{0}^{t} d t^{\prime} \gamma\left(t^{\prime}\right)\right)
$$


is amenable to a pure diffusion equation

$$
\frac{\partial c}{\partial \tau}=\frac{\partial^{2} c}{\partial \xi^{2}}
$$

In particular, if $c(x, t=0)=N \delta(x)$, then

$$
c(x, t)=\frac{N}{\sqrt{2 \pi \tau}} e^{-x^{2} / 2 \eta^{2}} .
$$

The scale over which the gradient of concentration $\left|\partial_{x} c\right|$ is localized is the Batchelor scale $\eta$. We have on purpose kept a general frame to allow for the stretching rate to be time dependent

$$
\frac{\gamma(t)}{\gamma}=\beta(\gamma t)^{\beta-1}
$$

with $\beta$ a number reflecting the accelerated nature of the stretch intensity $(\beta>1)$ or its slowing down $(\beta<1)$. The above formulation has no fundamental justification other than being easily adaptable to different flow configurations, knowing that, in nature, diverse behaviors exist concomitantly (see Sec. IV A). The corresponding Batchelor scale in Eq. (8) relaxes when $\gamma t \gtrsim 1$ towards (remember that $\int^{u} e^{2 t^{\beta}} d t \underset{u \gg 1}{\rightarrow} e^{2 u^{\beta}} / 2 \beta u^{\beta-1}$ for $\beta>1$ )

$$
\eta \sim \sqrt{\frac{D}{\beta \gamma}}(\gamma t)^{(1-\beta /) 2}
$$

and coincides with the usual constant value $\sqrt{D / \gamma}$ in exponentially diverging flows for $\beta=1$. The concentration gradient goes on steepening in the accelerated regions of the flow and relaxes for slowed down stretchings. There are thus three different characteristic length scales in this problem: the distance between two material points of the substrate in a direction transverse to the lamella given by $s_{0} \exp \left[-\int_{0}^{t} d t^{\prime} \gamma\left(t^{\prime}\right)\right]$, the width of the vapor concentration profile $\eta$, and the lamella thickness $s$, which is the thickness of the visible part of the spatial support where droplets have still not evaporated.

\section{B. Application to an evaporating strip}

The decay rate $\dot{s}$ of an evaporating lamella transverse thickness $s$ is the sum of two contributions: a kinematic contribution from the underlying flow compression $-\gamma(t) s$ and a contribution from the surface ablation of the lamella. By ablation we mean the disappearance of these droplets at the border of the lamella, which, because the vapor diffusion front has moved towards the dry environment, is no longer in equilibrium, and evaporates as explained in Sec. II C. Individual droplets evaporate in a typical short time $t_{1}$ once exposed to a dryer environment, so their evaporation location progresses over a (comparatively slow) time scale given by the speed of the vapor diffusion front itself. The ablative contribution to $\dot{s}$ thus corresponds to the mass loss $\rho \dot{s}$ given by the mass flux of vapor $M j$ diffusing away from the lamella, where $j=-\left.2 D \partial_{x} c\right|_{s / 2} \approx 2 D\left(c_{s}-c_{\infty}\right) / \eta$ is the vapor concentration diffusive flux leaving the lamella, the factor 2 standing for its two sides. The overall dynamics of the spray lamella thickness then is written as the sum of two velocities, with $v=M j / \rho$ the ablation velocity

$$
\begin{aligned}
& \dot{s}=-\gamma(t) s-v, \\
& v=\frac{\rho_{s}-\rho_{\infty}}{\rho} \frac{2 D}{\eta} .
\end{aligned}
$$

Setting $\mathcal{S}=s / s_{0}$ and $\mathcal{T}=\gamma t$ and owing to Eq. (12), we have

$$
\dot{\mathcal{S}}=-\beta \mathcal{T}^{\beta-1} \mathcal{S}-\phi \sqrt{\beta \mathcal{T}^{\beta-1}}
$$


with

$$
\phi=\frac{\rho_{s}-\rho_{\infty}}{\rho} \frac{2}{\sqrt{\mathrm{Pe}}},
$$

where the Péclet number Pe is given in Eq. (4).

In the absence of evaporation $(\phi=0)$, the lamella transverse thickness is slaved to the flow kinematics and relaxes as $e^{-\mathcal{T}^{\beta}}$. However, for $\phi>0$, the constant surface ablation velocity causes the thickness to vanish in a finite time. With $\beta=1$, for example, Eq. (15) amounts to $\dot{\mathcal{S}}=-\mathcal{S}-\phi$, which is solved

$$
\mathcal{S}=(1+\phi) e^{-\mathcal{T}}-\phi
$$

A lamella stretched at a constant rate $\gamma$ disappears when $s\left(t_{v}\right)=0$, defining its lifetime $t_{v}$ as

$$
t_{v}=\frac{1}{\gamma} \ln \left(1+\phi^{-1}\right) \text {. }
$$

For the same reason that both are ruled by a stretching enhanced diffusion process, the lifetime in Eq. (18) has the same structure as the mixing time $t_{m}$ in Eq. (4): It is proportional to the inverse of the deformation rate of the substrate $\gamma^{-1}$ and the diffusive (namely, $D$ ) and thermodynamic (namely, $\rho_{s}$ ) properties of the vapor as well as the composition of the initial spray (namely, $\rho$ ) only come into play as weak logarithmic corrections, unlike for the $d^{2}$ law. However, if the order of magnitude of $t_{v}$ is given by the flow kinematics, Eq. (18) consistently accounts for the fact that if $\rho_{\infty} \rightarrow \rho_{s}$, or $D \rightarrow 0$, the evaporation time is infinite (i.e., increases as $-\ln \phi$ when $\phi \rightarrow 0$ ), like for the $d^{2}$ law.

\section{RESULTS}

The evolution of a portion of the transitional $\left(\operatorname{Re} \approx 10^{3}\right)$ ethanol spray jet as it destabilizes in air is shown in Fig. 5(a), up to its disappearance. The core of the jet undergoes a sinusoidal destabilization due to shear with the ambient air and is stretched longitudinally. Among the many realizations of the same event in this flow, we have singled out and analyzed one for which the motion lies essentially in the visualization plane, with very little transverse displacement. This fact is established in the following way: The trajectories of the droplets in the spray are obtained by projecting their successive locations on a single frame [Fig. 5(b)], from which their velocity component $v_{\lambda}$ aligned with the direction $\lambda$ of the lamella support can be measured. This procedure identifies the stagnation point in $\lambda=0$ where $v_{\lambda=0}=0$. It is found that $v_{\lambda}$ is proportional to the distance $\lambda$ from the stagnation point, thus defining a stretching rate $\gamma$ such that $v_{\lambda}=\gamma \lambda$. The same measurement is repeated at three different instants of time in the course of the lamella life. Figure 5(c) shows that the three consecutive values of $\gamma \approx 15 \mathrm{~s}^{-1}$ are fairly identical, thus demonstrating that (i) the flow kinematics is of an exponentially diverging type and (ii) the stretching (compression) rate $\gamma$ is independent of time. The corresponding Péclet number $\mathrm{Pe} \approx 102$ is larger than unity, suggesting that the lamella width can be suitably described by Eq. (17).

Next we measure in the same experiment the transverse thickness $s(t)$ as a function of time, which, according to Eq. (17), should be tangential to $e^{-\gamma t}$ at short times if the motion were strictly two dimensional. We check in Fig. 5(d) that this is indeed the case, thus confirming that the initial decay rate of $s(t)$ is indeed attributable to a two-dimensional stretching protocol.

The overall trajectory of $s(t)$ is well described by Eq. (17), up to $s\left(t_{v}\right)=0$, thus legitimizing the representation we have made of the lamella width dynamics and of its lifetime $t_{v}$ in Eq. (18) in that particular instance where the stirring flow kinematics and the lamella width evolution can be documented independently. Also shown in Fig. 5(d) is the total width of the vapor thickness profile, which has decayed to reach the Batchelor scale $\sqrt{D / \gamma}$ by the time the spray thickness has vanished.

With the same ethanol spray, we further analyzed the dynamics and lifetimes of a collection of lamellae by varying the jet Reynolds number and measured concomitantly both the stretching rate $\gamma$ and the lifetime $t_{v}$ for each of them. The results reported in Fig. 6 suggest that Eq. (18) offers a 


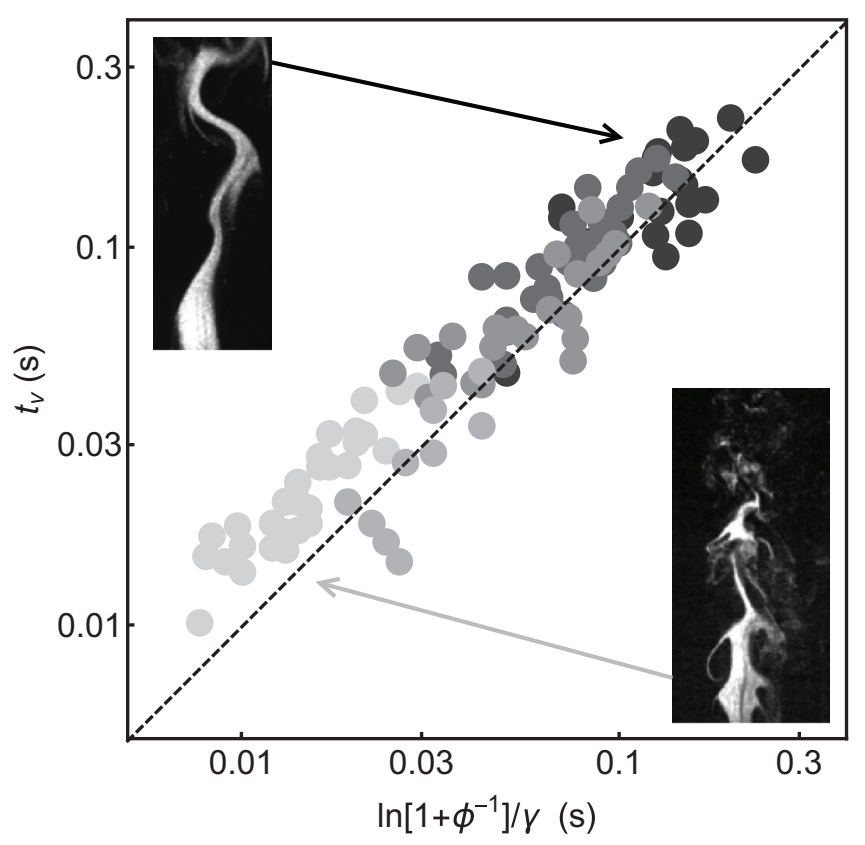

FIG. 6. Ethanol spray lamella measured lifetimes $t_{v}$ versus their expected value from Eq. (18) for various stretching rates $\gamma$ obtained at different jet Reynolds numbers for $10^{3} \lesssim \operatorname{Re} \lesssim 2 \times 10^{3}$. The inserts show examples of the fields from which the analyzed lamellae have been picked up.

quantitative description of the lifetime of a stretched lamella from a dense spray evaporating in a dry environment.

\section{A. Changing the stretching law}

In unstable or turbulent flows, stretching rates are broadly distributed in intensity. This is the reason for the existence of broad concentration distributions in dispersing scalar mixtures [35]. Not only is the intensity of the elongation distributed, but so are the stretching laws themselves: The Lagrangian histories among a set of lamellae are also diverse and, if the exponential stretching is the rule on average, exceptions do exist. We discuss two of them.

Occasionally, the stretching experienced by a lamellae may be superexponential, when the development of the shear instability is very sudden, as in Fig. 7(a). The same procedure as the one described before shows that the decay rate of the lamellae transverse thickness $\gamma(t)=-\dot{s} / s$ increases with time at short times. In our general ad hoc treatment of Sec. III, this corresponds to a case where $\beta>1$ and indeed the measured lamella thickness $s(t)$ decays faster than an exponential from the start, as shown in Fig. 8(a); we find $\beta=1.65$ in that case. Nevertheless, the overall trajectory of $s(t)$ is well represented by Eq. (15) down to its complete disappearance, which occurs consistently sooner than with an exponential stretch [see Fig. 5(d)]. The reason for this accelerated disappearance is that the ablation now intensifies in time because the Batchelor scale $\eta$ gets smaller as time proceeds when $\beta>1$ [see Eq. (12)], so vapor is transported faster away from the core of the lamella.

In contrast, the lamellae formed by the rolling-up vortices at the edge of the jet for a slightly higher Reynolds number exhibit a stretching rate decreasing in time [Fig. 7(b)]. It is well known that, in persistent vortices, material lines align with the streamlines, thus progressively canceling their rate of elongation [34]. In the terminology of Sec. III, this corresponds to $\beta<1$ and the measured lamella thickness $s(t)$ decay is indeed observed to slow down in Fig. 8(b); we find $\beta=0.65$ in that case. Since the Batchelor scale now gets thicker as time proceeds, the ablation velocity decays in 
(a)

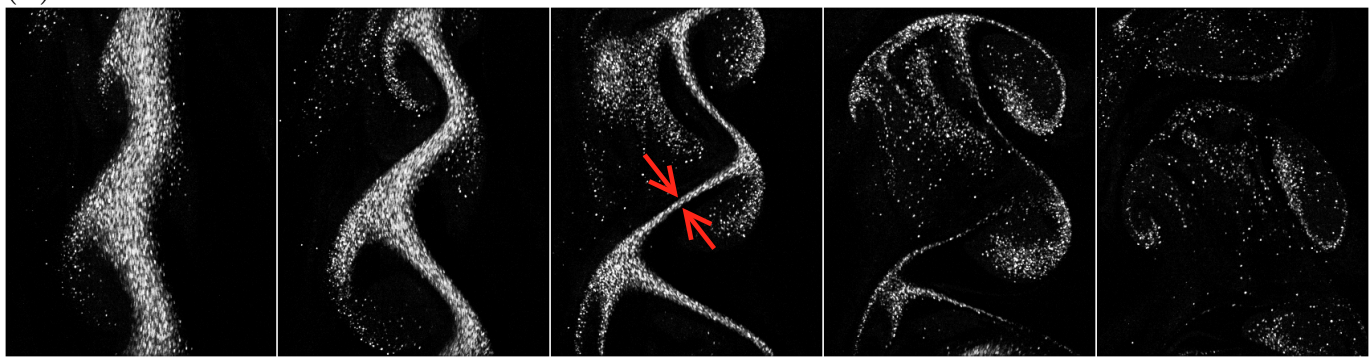

(b)
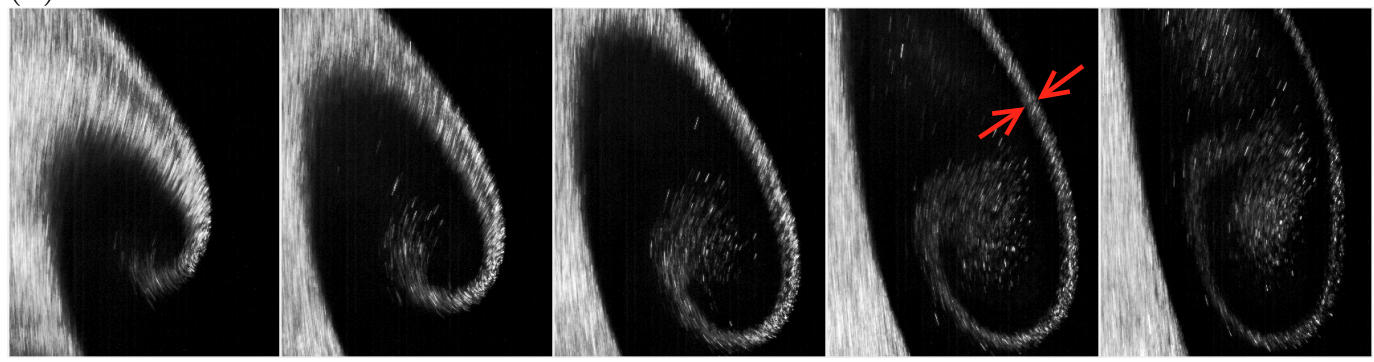

FIG. 7. Lamella evaporation of an ethanol dense spray with time-varying stretching rates. (a) Accelerated stretching for $\operatorname{Re} \approx 10^{3}$ and $s_{0}=8 \mathrm{~mm}$. Images are separated by $\Delta t=0.06 \mathrm{~s}$. (b) Slowed down stretching for $\operatorname{Re} \approx 2 \times 10^{3}$ and $s_{0}=5 \mathrm{~mm}$. Images are separated by $\Delta t=0.02 \mathrm{~s}$.

time, remaining however finite, thus ultimately leading to the complete disappearance of the lamella. This consistently occurs later than with an exponential stretch.

Altering the stretching kinematics does not change the evaporation mechanism of the lamella, which always results from surface ablation. It is the time dependence of the ablation velocity in Eq. (14), through the time dependence of the Batchelor scale in Eq. (12), that is a function of the stretching strength, or persistency, and therefore determines if the lamella life is shortened or extended.
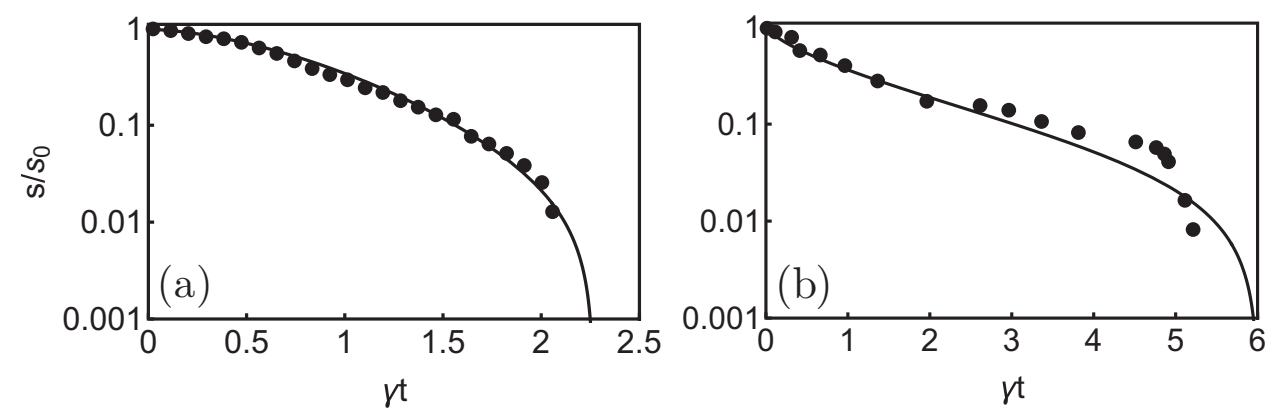

FIG. 8. Lamella thickness evolutions $s(t)$ normalized by their initial value $s_{0}$ for the (a) accelerated and slowed down stretchings in Fig. 7 for (a) $\gamma=9 \mathrm{~s}^{-1}, \mathrm{Pe}=40, \phi=0.034$, and $\beta=1.65$ giving $\gamma t_{v} \approx 2.25$ and (b) $\gamma=23 \mathrm{~s}^{-1}, \mathrm{Pe}=55, \phi=0.029$, and $\beta=0.65$ giving $\gamma t_{v} \approx 5-6$. The solid lines are the expected trajectories from Eq. (15) with the corresponding value of $\beta$ and $\phi$, to be compared with the exponential $(\beta=1)$ intermediate case in Fig. 5 for which $\gamma t_{v} \approx 4$. 


\section{B. How dense is a dense spray?}

Around an isolated droplet evaporating in an environment at rest and defining $\Delta \rho=\rho_{s}-\rho_{\infty}$, the vapor density decays in the radial direction $r$ as $\rho_{\infty}+\Delta \rho(2 d / r)$ for $d / 2 \ll \sqrt{D t}$, hence the $d^{2}$ law in Eq. (3) (see [36] for the analogous problem of gas bubbles dissolving in a liquid). The length-scale characteristic of the vapor density gradient is thus the droplet radius $d / 2$. Once the droplet has evaporated however, the vapor extends over an enrichment zone of radius $\ell_{e}$ such that $d^{3} \rho_{l} \sim \ell_{e}^{3} \Delta \rho$, that is,

$$
\ell_{e} \sim d\left(\frac{\rho_{l}}{\Delta \rho}\right)^{1 / 3} .
$$

Other droplets within this enrichment zone will have their own vapor profile smoothed and evaporation rate hindered by the nearby droplet(s). Therefore, a spray may be considered as dense and nonevaporating if the distance $\ell$ between the droplets is such that

$$
\ell \ll \ell_{e},
$$

the overlapping vapor density profiles from their neighbors preventing each individual droplet from evaporation. This is the limit in which we have conducted the present discussion, assuming that no evaporation at all was occurring in the core of a spray lamella. With ethanol, the distance $\ell_{e}$ is typically 20 times larger than $d$, of the same order, but nevertheless larger than the distance $\ell$ between the drops in the spray (Fig. 1), which is somewhat smaller than $10 d$.

In the bulk of a dense spray, incompressible deformations do not alter the droplet concentration field, which behaves as a passive scalar as long as St $\ll 1$. Although the droplet field is stretched and compressed, $\ell$ is conserved, in the mean. However, at the end of the evaporation process of a lamella, when its transverse thickness is ultimately composed of a unique string of droplets moving away from each other under the action of the stretch, the condition (20) is no longer fulfilled, even if it was initially true in the original spray. The idealized representation of a lamella saturated in vapor is no longer valid, giving way to the isolated droplet limit. These very last distant droplets evaporate on the short time scale $t_{1}$ given by the $d^{2}$ law in Eq. (3), explaining probably why the final experimental trajectories of $s(t)$ shrink to zero systematically faster than expected from the diffuse lamella model (see Figs. 5 and 8). The lamella evaporation time (for $\beta=1$ ) in Eq. (18) is thus meaningful provided it is longer than the time $\gamma^{-1} \ln \left(s_{0} / \ell\right)$ taken by the kinematics of the flow to bring the lamella down to a unique string of droplets, meaning its width down to $\ell$, which is for

$$
\ell<\phi s_{0},
$$

a condition marginally met in the present experiments.

Finally, for the one-dimensional diffusion (6) to be a fair caricature, the interface between the spray lamella and the diluting medium must not be too rough. For this, the thickness of the vapor gradient at the lamella interface must be larger than the length scale of the maximal irregularity in the fine-grained vapor density profile in the spray, given by $\ell_{e}$. The lamella border can be considered as smooth as long as $\ell_{e}<\sqrt{D / \gamma}$, that is,

$$
\frac{d}{s_{0}}<\frac{\left(\Delta \rho / \rho_{l}\right)^{1 / 3}}{\sqrt{P e}},
$$

a condition, with $\mathrm{Pe}=O\left(10^{2}\right)$ and $d / s_{0}=O\left(10^{-3}\right)$, always fulfilled by far in the present conditions.

\section{CONCLUSION}

We have shown that, at least when they are sufficiently close to each other and small, the way a collection of nearby droplets evaporate in a dry stirred environment can be understood from the dynamics of a crucial although invisible partner of the phenomenon, namely, the vapor concentration field. The support of the droplet field is stirred as a nondiffusive tracer, while the interstitial vapor, 
saturated in the dense regions of the spray, diffuses like a passive scalar would do, at a rate enhanced by the intensity of the underlying stretching motions. In this respect, the lamellar representation of the mixture has been useful at predicting quantitatively the fate of the spray and in particular its lifetime $t_{v}$. This lifetime (18) scales as the inverse of the deformation rate of the stirring flow $\gamma^{-1}$ and reflects, like a mixing time (4), the time it takes for the vapor concentration differences between the inside and the outside of the spray to vanish, thus allowing the droplets to be exposed to the dry environment where they finally evaporate, on the much shorter time scale $t_{1}$ of the $d^{2}$ law (3). Although both result from a diffusion process, $t_{1}$ involves the droplet diameter $d$, while $t_{v}$ involves the initial transverse size of the stretched spray lamella $s_{0}$ as a whole. Because of this scale separation, $t_{v}$ is larger than $t_{1}$ in usual ambient humidity conditions at moderate Péclet number (see, e.g., Sec. II C)

$$
\begin{aligned}
\frac{t_{v}}{t_{1}} & \sim\left(\frac{s_{0}}{d}\right)^{2} \frac{\Delta \rho}{\rho_{l}} \frac{\ln \mathrm{Pe}}{\mathrm{Pe}} \\
& \gg 1 \text { (typically) } .
\end{aligned}
$$

In the present experiments, the Péclet number should be as large as $10^{4}$ for the two times to be equal, while we rather have $\mathrm{Pe}=O\left(10-10^{2}\right)$.

The caricature we have draw here provides convincing predictions for a unique spray lamella stretched in an infinitely extended dry environment. We know, however, from the scalar mixing context that this idealization is limited to dispersing mixtures that can be approximated as a set of noninteracting lamellae only, while confined mixtures behave in a completely different way: In that other ideal limit, the interaction between the lamellae is the primary ingredient constructing the scalar fluctuations [37-39]. In addition, the nature of the interaction is, in a self-similar fashion, of a very similar type as the one between individual droplets in a dense spray: Their diffusive boundaries overlap, thus slowing down the concentration decay. This is what happens when the

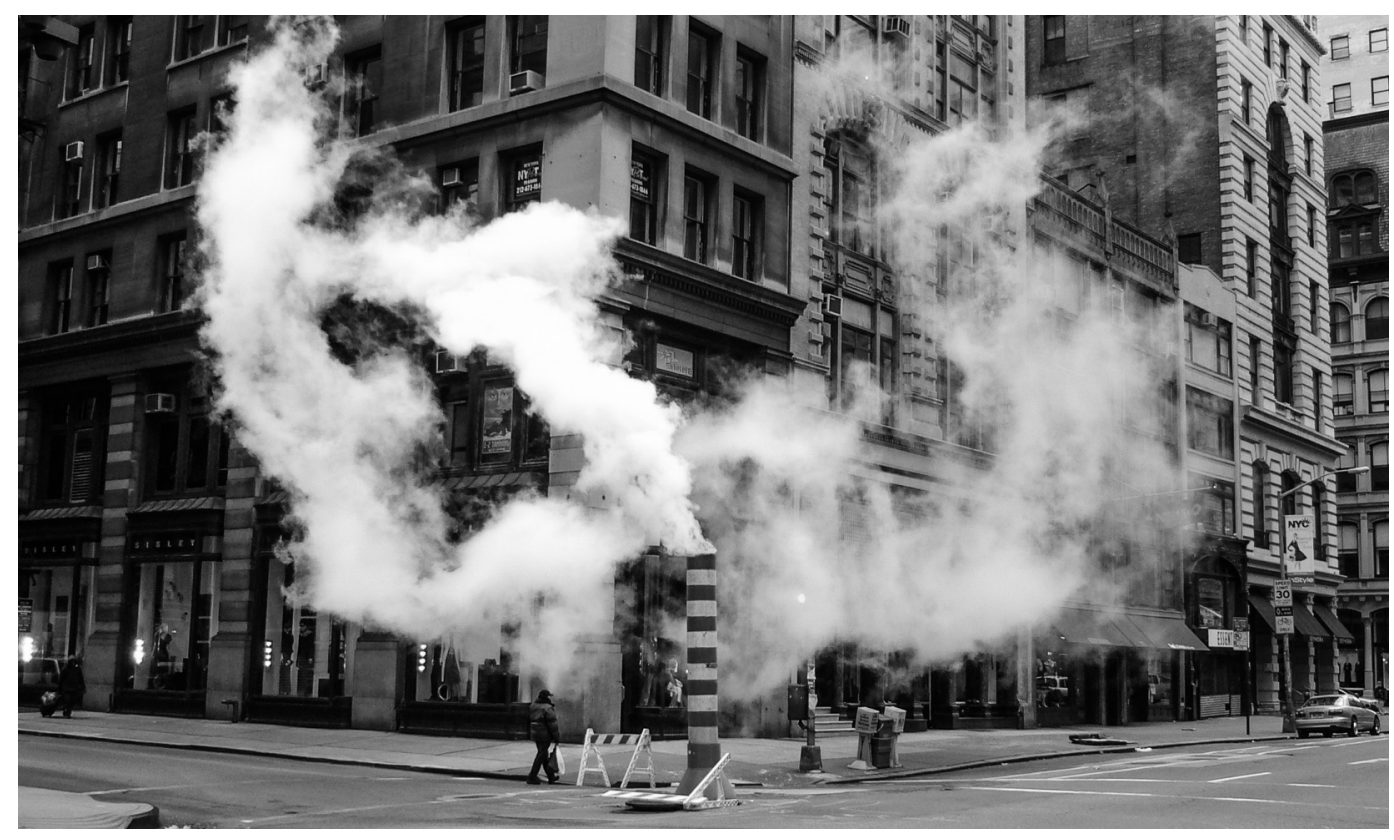

FIG. 9. Water spray plume from one of the familiar steam exhaust pipes in New York City on a windy Thanksgiving day. The plume is chopped off in many elementary stretched lamellae, which, however, cannot evaporate before the plume has broadened to incorporate enough dry outside air. Credit: picture by E. Villermaux. 
spray lamellae produced by the stirring motions are themselves dense in space. In this instance, the evaporation of the spray does not depend on molecular diffusion or on the stirring strength at the lamella scale, but on the ability of the flow to incorporate the dry environment and put it in contact with the spray lamellae. The limiting factor setting the spray lifetime is in that case the large-scale entrainment property of the flow [40], which relies on its (often turbulent) dispersion properties. Figure 9 illustrates well this effect prevailing in many applications, from agricultural spraying and the notorious wind-induced spray drift problem, to decease transmission by human exhalations [6], a subject for future research.

\section{ACKNOWLEDGMENTS}

We thank D. Durox for introducing us to the IPI technique, P. Meunier for advice, and the Agence Nationale de la Recherche, France (Grant No. ANR-14-CE35-0031-01) for funding of the ANR-DFG TurbMix.

[1] B. J. Mason, The Physics of Clouds (Clarendon, Oxford, 1971).

[2] H. R. Pruppacher and J. D. Klett, Microphysics of Clouds and Precipitation (Kluwer, Dordrecht, 1997).

[3] F. Veron, C. Hopkins, E. L. Harrison, and J. A. Mueller, Sea spray spume droplet production in high wind speeds, Geophys. Res. Lett. 39, L16602 (2012).

[4] N. Ashgriz, Handbook of Atomization and Sprays (Springer, New York, 2011).

[5] G. J. Dorr, A. J. Hewitt, S. W. Adkins, J. Hanan, H. Zhang, and B. Noller, A comparison of initial spray characteristics produced by agricultural nozzles, Crop Prot. 53, 109 (2013).

[6] L. Bourouiba, E. Dehandschoewercker, and J. W. M. Bush, Violent expiratory events: On coughing and sneezing, J. Fluid Mech. 745, 537 (2014).

[7] J. Bellan and R. Cuffel, A theory of nondilute spray evaporation based upon multiple drop interactions, Combust. Flames 51, 55 (1983).

[8] N. Chigier, Group combustion models and laser diagnostic methods in sprays: A review, Combust. Flames 51, 127 (1983).

[9] C. Bourgès-Monnier and M. E. R. Shanahan, Influence of evaporation on contact angle, Langmuir 11, 2820 (1995).

[10] N. Shahidzadeh-Bonn, S. Rafaï, A. Azouni, and D. Bonn, Evaporating droplets, J. Fluid Mech. 549, 307 (2006).

[11] A. M. Cazabat and G. Guéna, Evaporation of macroscopic sessile droplets, Soft Matter 6, 2591 (2010).

[12] I. Langmuir, The evporation of small spheres, Phys. Rev. 12, 368 (1918).

[13] B. Topley and T. Whytlaw-Gray, Experiments on the rate of evaporation of small spheres as a method of determining diffusion coefficients, Philos. Mag. 4, 873 (1927).

[14] H. G. Houghton, A study of the evaporation of small water drops, J. Appl. Phys. 4, 419 (1933).

[15] J. K. Hardy, Evaporation of drops of liquid, Aeronautical Research Council, Reports and Memoranda, Ministry of Supply Report No. 2805, 1947 (unpublished).

[16] D. B. Spalding, Combustion of fuel particles, Fuel 1, 121 (1951).

[17] H. W. Morse, On evaporation from the surface of a solid sphere, Proc. Am. Acad. Arts Sci. 45, 361 (1910).

[18] W. E. Ranz and W. R. Marshall, Evaporation from drops, Part I, Chem. Eng. Prog. 48, 141 (1952).

[19] W. E. Ranz and W. R. Marshall, Evaporation from drops, Part II, Chem. Eng. Prog. 48, 173 (1952).

[20] P. Jenny, D. Roekaerts, and N. Beishuizen, Modeling of turbulent dilute spray combustion, Prog. Energ. Combust. Sci. 38, 846 (2012).

[21] A. L. Sanchez, J. Urzay, and A. Linan, The role of separation of scales in the description of spray combustion, Proc. Combust. Inst. 35, 1549 (2015).

[22] J. K. Eaton and J. R. Fessler, Preferential concentration of particles by turbulence, Int. J. Multiphase Flow 20, 169 (1994). 
[23] G. Falkovich, A. Fouxon, and M. G. Stepanov, Acceleration of rain initiation by turbulence, Nature (London) 419, 151 (2002).

[24] J. Bec, Multifractal concentrations of inertial particles in smooth random flows, J. Fluid Mech. 528, 255 (2005).

[25] W. E. Ranz, Application of a stretch model to mixing, diffusion and reaction in laminar and turbulent flows, AIChE J. 25, 41 (1979).

[26] E. Villermaux, On dissipation in stirred mixtures, Adv. Appl. Mech. 45, 91 (2012).

[27] H. A. Becker and T. A. Massaro, Vortex evolution in a round jet, J. Fluid Mech. 31, 435 (1968).

[28] S. P. Burke and T. E. W. Schumann, Diffusion flames, Ind. Eng. Chem. 20, 998 (1928).

[29] E. Villermaux and D. Durox, On the physics of jet diffusion flames, Combust. Sci. Technol. 84, 279 (1992).

[30] C. Tropea, Optical particle characterization in flows, Annu. Rev. Fluid Mech. 43, 399 (2011).

[31] H. Bocanegra Evans, N. Dam, D. van der Voort, G. Bertens, and W. van de Water, Measuring droplet size distributions from overlapping interferometric particle images, Rev. Sci. Instrum. 86, 023709 (2015).

[32] G. Guéna, J. Corde, S. Fouilloux, J.-B. d'Espinose, F. Lequeux, and L. Talini, Evaporation of an emulsion trapped in a yield stress fluid, Eur. Phys. J. E 28, 463 (2009).

[33] G. K. Batchelor, Small-scale variation of convected quantities like temperature in turbulent fluid. Part 1. General discussion and the case of small conductivity, J. Fluid Mech. 5, 113 (1959).

[34] P. Meunier and E. Villermaux, How vortices mix, J. Fluid Mech. 476, 213 (2003).

[35] J. Duplat, C. Innocenti, and E. Villermaux, A nonsequential turbulent mixing process, Phys. Fluids 22, 035104 (2010).

[36] P. S. Epstein and M. S. Plesset, On the stability of gas bubbles in liquid-gas solutions, J. Chem. Phys. 18, 1505 (1950).

[37] E. Villermaux and J. Duplat, Mixing as an Aggregation Process, Phys. Rev. Lett. 91, 184501 (2003).

[38] J. Duplat and E. Villermaux, Mixing by random stirring in confined mixtures, J. Fluid Mech. 617, 51 (2008).

[39] T. Le Borgne, M. Dentz, and E. Villermaux, The lamellar description of mixing in porous media, J. Fluid Mech. 770, 458 (2015).

[40] W. J. A. Dahm and P. E. Dimotakis, Meausurements of entertainment and mixing in turbulent jets, AIAA J. 25, 1216 (1987). 